\title{
Quasar evolution: black hole mass and accretion rate determination
}

\author{
Deborah Dultzin-Hacyan, ${ }^{1}$ Paola Marziani, ${ }^{2}$ C. Alenka Negrete ${ }^{1}$ \\ and Jack W. Sulentic ${ }^{3}$ \\ ${ }^{1}$ Instituto de Astronomía, UNAM, Apdo. Postal 70-264, 04510 Mexico D.F., Mexico \\ 2 INAF, Osservatorio Astronomico di Padova, Vicolo dell'Osservatorio 5, I-35122 Padova, Italy \\ ${ }^{3}$ Department of Physics and Astronomy, University of Alabama, Tuscaloosa, AL 35487, USA \\ email: deborah@astroscu.unam.mx, paola.marziani@oapd.inaf.it, giacomo@merlot.astr.ua.edu
}

\begin{abstract}
Accurate measurements of emission line properties are crucial to understand the physics of the broad line region in quasars. This region consists of warm gas that is closest to the quasar central engine and has not been spatially resolved for almost all sources. We present here an analysis of optical and IR data for a large sample of quasars, covering the $\mathrm{HI} \mathrm{H} \beta$ spectral region in the redshift range $0 \lesssim z \lesssim 2.5$. Spectra were interpreted within the framework of the the so-called "eigenvector 1" parameter space, which can be viewed as a tentative H-R diagram for quasars. We stress the lack of spectral evolution in the low ionization lines of quasars, with prominent FeII emission also at $z \gtrsim 2$. We also show how selection effects influence the ability to find quasars radiating at low Eddington ratio in flux-limited surveys. The quasar similarity at different redshift is probably due to the absence of super-Eddington radiators (at least within the caveats of black hole mass and Eddington ratio determination discussed in this paper) as well as to the limited Eddington ratio range within which quasars seem to radiate.
\end{abstract}

Keywords. Quasars: general - quasars: emission lines

\section{Introduction}

The current inability to spatially resolve the central regions of quasars is being circumvented by several alternative approaches. Results from reverberation mapping of NGC 5548 (Peterson et al. 2002) with HST and ground monitoring of broad line response to UV continuum variations suggest that high ionization lines (HILs) respond within a few days, while low ionization lines (LILs) respond within a month. By now more than 30 Seyfert nuclei have been studied in this way (Peterson et al. 2004). An important result is that the response time (proportional to the size of the emitting region) is correlated to luminosity (Kaspi et al. 2005): $r_{\mathrm{BLR}} \propto L^{\alpha}$. This relationship has been exploited to compute the central black hole mass $M_{\mathrm{BH}}$ in the absence of reverberation mapping data, under the assumption that the bulk line-emitting gas dynamics is virial. In this case one can apply the relationship $M_{\mathrm{BH}}=f \cdot G \cdot \mathrm{FWHM}^{2} / r_{\mathrm{BLR}}$, where $G$ is the gravitational constant, FWHM the full-width at half maximum of a suitable emission line, and $f$ a factor that depends on the broad line emitting region (BLR) structure and viewing angle. There are several caveats with this approach (reviewed by (Marziani et al. 2006; see also Sulentic et al. 2006). First, the $r_{\mathrm{BLR}}-L$ relationship is not free from uncertainties. Reverberation mapping-based determinations of $r_{\mathrm{BLR}}$ are affected by the non-negligible radial extent of the optically thick BLR. The derived $r_{\mathrm{BLR}}$ is not a very well defined quantity. The luminosity index $\alpha$ is also somewhat uncertain because of the intrinsic scatter in the correlation and lack of sampling at high luminosity. However, the main issue is that a good understanding of the structure and dynamics of BLR is required to 
justify the virial assumption. This knowledge is still lacking, and Principal Component Analysis (PCA) of quasars samples raise sources of concerns.

\section{The eigenvector 1 of AGN}

The low-ionization line properties of quasars can be organized along an "eigenvector 1" sequence, mainly governed by Eddington ratio, but also affected (to a second-order degree) by the angle at which the BLR are observed, and $M_{\mathrm{BH}}$ (still more speculative sources of scatter are iron abundances and black hole spin). On an optical plane defined by the intensity ratio $R_{\mathrm{Fe}}$ between the FeII blend centered at $4570 \AA$ and the broad component of $\mathrm{H} \beta\left(\mathrm{H} \beta_{\mathrm{BC}}\right)$, and by the FWHM of $\mathrm{H} \beta_{\mathrm{BC}}$ itself, sources are distributed along an elbow sequence: almost all objects show $R_{\mathrm{Fe}} \lesssim 0.5$ if $\mathrm{FWHM}\left(\mathrm{H} \beta_{\mathrm{BC}}\right) \gtrsim 4000$ $\mathrm{km} \mathrm{s}^{-1}$. We find most radio-loud quasars toward the distribution extreme with lowest $R_{\mathrm{Fe}}$ and broadest $\mathrm{H} \beta$, where we also observe a rather high ionization spectrum, with prominent [OIII] $\lambda \lambda 4959,5007$ lines. The median $\mathrm{H} \beta_{\mathrm{BC}}$ profiles are deformed Gaussians with red-ward asymmetries. Toward the highest $R_{\mathrm{Fe}}$, narrowest $\mathrm{H} \beta_{\mathrm{BC}}$ end of the diagram, sources show a rather low-ionization spectrum, and less prominent [OIII] $\lambda \lambda 44959,5007$ emission. The $\mathrm{H} \beta_{\mathrm{BC}}$ profiles are most often of Lorentzian shape, sometimes with a blueward asymmetry. The prototypical HIL CIV $\lambda 1549$ shows large blue-shifts with respect to the quasar reference frame. These observational constraints suggest that in the latter sources (which include Narrow Line Seyfert 1s but also objects with $\operatorname{FWHM}\left(\mathrm{H} \beta_{\mathrm{BC}}\right) \lesssim$ $4000 \mathrm{kms}^{-1}$, collectively referred to as "Population A"). the LIL and HIL emitting regions obey to different kinematics: while the mostly symmetric profiles of $\mathrm{H} \beta_{\mathrm{BC}}$ are naïvely consistent with virial motion, most CIV $\lambda 1549$ profiles are not.

\section{High- $z$ quasars $M_{\mathrm{BH}}$ and Eddington ratio determination}

The HIL CIV $\lambda 1549$ has been the line of choice for black hole mass estimation in high$z$ quasars (see the careful analysis by Vestergaard \& Peterson(2006)), although it is in principle unsuitable for at least two reasons: (1) blue-ward asymmetric profiles, or even centroid blue-shifts (up to several thousands $\mathrm{km} \mathrm{s}^{-1}$ ) with respect to the best estimates of the rest frame of the source and (2) FWHM CIV $\lambda 1549$ does not correlate strongly with $\operatorname{FWHM}\left(\mathrm{H} \beta_{\mathrm{BC}}\right)$ which is the line of choice for low-redshift $M_{\mathrm{BH}}$ estimation. We made several attempts to deduce a correction for the CIV $\lambda 1549$-derived $M_{\mathrm{BH}}$ from properties intrinsic to the CIV $\lambda 1549$ profile shape (i.e., width, asymmetry and kurtosis), but we were unable to find an effective relationship. Blue-shifted CIV $\lambda 1549$ profiles are thought to arise in a high ionization wind resulting in a radial flow velocity that is not negligible relative to any rotational component. Our fears about CIV $\lambda 1549$-derived $M_{\mathrm{BH}}$ motivated us to pursue $\mathrm{H} \beta$ observations to the highest possible redshift.

The Balmer lines do not usually show very large line shifts although profiles can be very asymmetric. Both red and blue asymmetries are observed for $\mathrm{H} \beta_{\mathrm{BC}}$ which is the most studied line because it is relatively un-blended and is observable with optical spectrometers up to $z \approx 1$. The most ambiguous sources from the point of view of $M_{\mathrm{BH}}$ are those with $\operatorname{FWHM}\left(\mathrm{H} \beta_{\mathrm{BC}}\right) \gtrsim 4000 \mathrm{~km} \mathrm{~s}^{-1}$ (Population B), and redshifted profiles and/or red asymmetries. In this case, not all parts of the $\mathrm{H} \beta$ profile respond to continuum changes in the same way implying that some of the line emitting gas may be optically thin or, less likely, not exposed to the variable Hi ionizing radiation. A strong BLR response to continuum fluctuations, coupled with a weak or absent response of the broader optically thin component, can lead to an overestimate of FWHM for the virialized BLR component resulting in an overestimate of $M_{\mathrm{BH}}$. For sources with $\mathrm{FWHM}\left(\mathrm{H} \beta_{\mathrm{BC}}\right) \lesssim 4000 \mathrm{~km} \mathrm{~s}^{-1}$, 

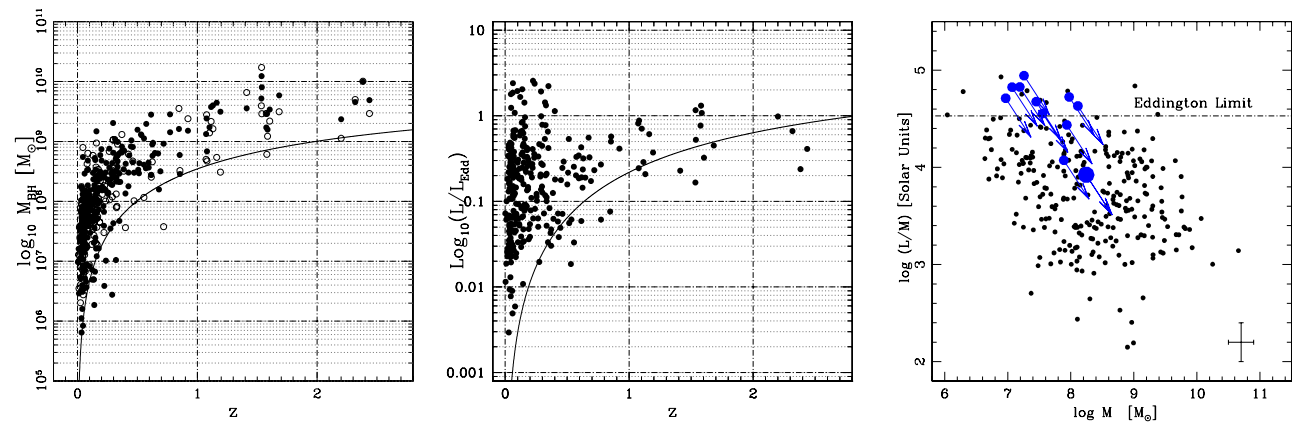

Figure 1. Left: Behavior of black hole mass as a function of $z$. Filled circles represent $M_{\mathrm{BH}}$ computed from corrected $\mathrm{FWHM}\left(\mathrm{H} \beta_{\mathrm{BC}}\right)$ values, while open circles from $\operatorname{FWHM}(\mathrm{FeII} \lambda 4570$. Center: Eddington ratio as a function of $z$ for calculations based on corrected $\operatorname{FWHM}\left(\mathrm{H} \beta_{\mathrm{BC}}\right)$ values. Filled lines indicate minimum $M_{\mathrm{BH}}$ (for Eddington ratio equal 1) and minimum Eddington ratio (for a "maximum" $M_{\mathrm{BH}} \approx 4 \cdot 10^{9} \mathrm{M}_{\odot}$ ) for a flux limited survey with $m_{\mathrm{B}} \approx 16.5$. Right: Super Eddington radiators may be due mainly to orientation effects. The larger circles indicate the so-called blue "outliers" which are sources believed to be viewed almost along the accretion disk axis.

the $\mathrm{H} \beta_{\mathrm{BC}}$ profile is usually well fit with a symmetric function and the BLR emission is thought to arise from a Keplerian disk. The most unreliable Pop. A sources, in a disk emission scenario, should be those observed near face-on where the rotational (i.e. virial) contribution to $\mathrm{FWHM}\left(\mathrm{H} \beta_{\mathrm{BC}}\right)$ is minimal. At least some of the face-on sources may be identified as the so-called "blue outliers" which show a weak and significantly blue-shifted [OIII] $\lambda \lambda 4959,5007$ lines (Collin et al. 2006).

A physical approach to $M_{\mathrm{BH}}$ estimation uses the FWHM of the variable part of the $\mathrm{H} \beta_{\mathrm{BC}}$ profile Peterson et al. (2004). LILs like MgII and FeII may yield more reliable results than $\mathrm{H} \beta_{\mathrm{BC}}$. Hi Balmer line emission can be substantial from gas in a variety of physical conditions. A correction can be derived only at low- $z$, from objects for which a proper observation of the variable part (rms) of the $\mathrm{H} \beta_{\mathrm{BC}}$ is possible. The optically thick BLR gas is responding to continuum changes shows a velocity dispersion correlated with - but slightly lower than that of the integrated profile at all FWHM. The reverberating part of $\mathrm{H} \beta_{\mathrm{BC}}$ and FeII provides width estimates which are consistent, as expected since they may be emitted in a similar sub-region within the BLR. Similar considerations apply to MgII $\lambda 2800$. Given measurement difficulties, and doubts about the virial assumption for most other lines, corrected measures for $\mathrm{H} \beta_{\mathrm{BC}}$, FeII, and $\mathrm{MgII} \lambda 2800$ may offer the best hope for reliable $M_{\mathrm{BH}}$ and Eddington ratio estimates out to $z \approx 2.5$.

\section{Quasar evolution: $M_{\mathrm{BH}}$ and Eddington ratio}

The E1 sequence has been interpreted as an evolutionary sequence. Young AGNs still possess a compact NLR; they may have plenty of fuel and radiate close to the Eddington limit and even show spectral evidence of circum-nuclear Starbursts. More massive, evolved systems may have less fuel and have had all the time to ionize interstellar clouds in the bulge of their host galaxies as well as to produce radio lobes exceeding the host galaxy size. These conclusions, however preliminary they may be, are inferred from the diversity of properties of low- $z$ quasar samples. To extend our observations to higher redshift, we observed 50 quasars in the range $1 \lesssim z \lesssim 2.5$ with the IR spectrometer ISAAC on the ESO VLT (30 published in Sulentic et al. 2004, 2006). The passable S/N $(\approx 30)$ and resolution allowed us to apply the same data analysis technique employed for about 
300 spectra from optical spectrometers (provided by Marziani et al. 2003). Our VLT spectra confirm that any dependence of $\operatorname{FWHM}\left(\mathrm{H} \beta_{\mathrm{BC}}\right)$ on source luminosity is weak. Low redshift trends for asymmetries and line shifts are preserved in the intermediate $z$ sample. It is therefore not certain that $\operatorname{FWHM}\left(\mathrm{H} \beta_{\mathrm{BC}}\right)$ is a valid estimator of the virial velocity for all sources even after proper [OIII] $\lambda, \lambda 4959,5007$ and FeII subtraction. We attempted to reconduct the observed $\mathrm{H} \beta_{\mathrm{BC}}$ FWHM to the FWHM of the optically thick reverberating $\mathrm{H} \beta_{\mathrm{BC}}$ sub-component as discussed earlier. The application of a correction largely removes the need of $M_{\mathrm{BH}} \gtrsim 10^{10} \mathrm{M}_{\odot}$, and produces a trend consistent with the one obtained from FeII width. There seems to be a limit to $M_{\mathrm{BH}}$ growth with $z$ for masses determined from virialization assumption for Balmer and FeII emitting gas at low and intermediate redshifts. This is reasonable because, if the dispersion velocity - bulge mass - $M_{\mathrm{BH}}$ relation holds for intermediate and high $z, M_{\mathrm{BH}} \gtrsim 5 \cdot 10^{9} \mathrm{M}_{\odot}$ would imply bulge velocity dispersions in excess of $700 \mathrm{~km} \mathrm{~s}^{-1}$ and bulge masses $\sim 10^{13} M_{\odot}$ which are not observed at low $z$ (Bernardi et al. 2005).

Perhaps surprisingly, apparent super-Eddington radiators seem to be confined at low $z$. If our interpretation is correct, these sources are viewed with their accretion disk almost pole-on; their $M_{\mathrm{BH}}$ may be severely underestimated. If a tentative orientation correction is applied, even these sources could radiate close but below the Eddington Limit (Fig. 1, right panel). It is interesting to note that the curve shown in Fig. 1 (center panel) seem to envelop nicely the observations, suggesting that, in a flux limited sample (here a limiting magnitude $m_{\mathrm{B}} \approx 16.5$ is assumed), we are starting to increasingly miss radiators above 0.01 the Eddington limit at $z \approx 0.3$, and that at $z \approx 1$ we may miss a significant population of low Eddington ratio radiators well sampled at low $z$ (mainly Population B sources which, in general, show smaller Civ $\lambda 1549$ blue-shifts).

\section{Conclusions}

Unfortunately, observing $\mathrm{H} \beta$ in the IR and even obtaining a reliable recessional velocity for the rest frame remain a challenge. However, it is important to increase the sample of observed objects to confirm the findings about the lack of very large masses $M_{\mathrm{BH}} \gtrsim 5 \cdot 10^{9}$ $\mathrm{M}_{\odot}$ and super-Eddington radiators, as well as to recover information on the evolution of Eddington ratio with $z$.

\section{References}

Bernardi M., Sheth R. K., Nichol R. C., Schneider D. P. \& Brinkmann J. 2005, AJ, 129, 61 Collin S., Kawaguchi T., Peterson B. M. \& Vestergaard M. 2006, A\&A, 456, 75

Kaspi S., Maoz D., Netzer H., Peterson B. M., Vestergaard M. \& Jannuzi B. T. 2005, ApJ, 629, 61

Marziani P., Dultzin-Hacyan D. \& Sulentic J. W. 2006, astro-ph/0606678

Marziani P., Zamanov R. K., Sulentic J. W. \& Calvani M. 2003, MNRAS, 345, 1133

Peterson B. M. et al. 2002, ApJ, 581, 197

Peterson B. M. et al. 2004, ApJ, 613, 682

Sulentic J. W., Repetto P., Stirpe G. M., Marziani P., Dultzin-Hacyan D. \& Calvani, M. 2006, A\&A, 456, 929

Sulentic J. W., Stirpe G. M., Marziani P., Zamanov R., Calvani M. \& Braito V. 2004, A\&A, 423,121

Vestergaard M., Peterson B. M. 2006, ApJ, 641, 689 